\title{
CDISC Alzheimer's Therapeutic Area User Guide Version 1.0
}

National Cancer Institute

\section{Source}

National Cancer Institute. CDISC Alzheimer's Therapeutic Area User Guide Version 1.0. NCI Thesaurus. Code C161458.

The 1.0 version of the CDISC Alzheimer's therapeutic area user guide. 